Homology, Homotopy and Applications, vol.12(1), 2010, pp.1-10

\title{
THE GLUING PROBLEM DOES NOT FOLLOW FROM HOMOLOGICAL PROPERTIES OF $\Delta_{p}(G)$
}

\author{
ASSAF LIBMAN \\ (communicated by J. P. C. Greenlees)
}

\begin{abstract}
Given a block $b$ in $k G$ where $k$ is an algebraically closed field of characteristic $p$, there are classes $\alpha_{Q} \in H^{2}\left(\operatorname{Aut}_{\mathcal{F}}(Q) ; k^{\times}\right)$, constructed by Külshammer and Puig, where $\mathcal{F}$ is the fusion system associated to $b$ and $Q$ is an $\mathcal{F}$-centric subgroup. The gluing problem in $\mathcal{F}$ has a solution if these classes are the restriction of a class $\alpha \in H^{2}\left(\mathcal{F}^{c} ; k^{\times}\right)$. Linckelmann showed that a solution to the gluing problem gives rise to a reformulation of Alperin's weight conjecture. He then showed that the gluing problem has a solution if for every finite group $G$, the equivariant Bredon cohomology group $H_{G}^{1}\left(\left|\Delta_{p}(G)\right| ; \mathcal{A}^{1}\right)$ vanishes, where $\left|\Delta_{p}(G)\right|$ is the simplicial complex of the non-trivial $p$-subgroups of $G$ and $\mathcal{A}^{1}$ is the coefficient functor $G / H \mapsto \operatorname{Hom}\left(H, k^{\times}\right)$. The purpose of this note is to show that this group does not vanish if $G=\Sigma_{p^{2}}$ where $p \geqslant 5$.
\end{abstract}

\section{Introduction}

Given a functor $M: \mathcal{C} \rightarrow \mathbf{A b}$, where $\mathcal{C}$ is a small category, we will write $H^{*}(\mathcal{C} ; M)$ for the groups $\lim _{\mathcal{C}}^{*} M$. When $\mathcal{C}$ has one object with a group $G$ of automorphisms, a functor $M: \mathcal{C} \rightarrow \mathbf{A b}$ is the same thing as a $G$-module and $H^{*}(G ; M) \cong \varliminf_{\mathcal{C}}^{*} M$.

Let us now fix a prime $p$ and let $\mathcal{F}$ be the fusion system of a block $b$ of a finite group $G$. As usual, we will write $\mathcal{F}^{c}$ for the full subcategory generated by the $\mathcal{F}$ centric subgroups in $\mathcal{F}$. Let $k$ be an algebraically closed field of characteristic $p$. In [8] Külshammer and Puig show that for every $\mathcal{F}$-centric subgroup $Q$ there is a canonically chosen class $\alpha_{Q} \in H^{2}\left(\operatorname{Aut}_{\mathcal{F}}(Q) ; k^{\times}\right)$. We view $\operatorname{Aut}_{\mathcal{F}}(Q)$ as a full subcategory of $\mathcal{F}^{c}$ and say that the gluing problem has a solution in $\mathcal{F}$ if there exists a class $\alpha \in H^{2}\left(\mathcal{F}^{c} ; k^{\times}\right)$, where $k^{\times}$is the constant functor, such that the restriction $\left.\alpha\right|_{\operatorname{Aut}_{\mathcal{F}}(Q)}$ is equal to $\alpha_{Q}$ for all $Q \in \mathcal{F}^{c}$.

Linckelmann showed in [10] that if the gluing problem has a solution in the fusion systems of all blocks then Alperin's weight conjecture is logically equivalent to a relation between the number $\mathbf{k}(b)$ of complex representations of $G$ associated to $b$ by

Received May 27, 2009, revised October 19, 2009; published on February 18, 2010.

2000 Mathematics Subject Classification: 20C20, 55N25, 05E25.

Key words and phrases: gluing problem, Alperin's conjecture, equivariant cohomology.

This article is available at http://intlpress.com/HHA/v12/n1/a1

Copyright (c) 2010, International Press. Permission to copy for private use granted. 
Knörr and Robinson [7] and the Euler characteristic of a certain cochain complex built from the fusion system of $b$ and the cohomology class $\alpha$.

Let $G$ be a finite group and $\mathcal{C}$ a finite $G$-poset. The (combinatorial) simplicial complex associated to $\mathcal{C}$, see [13, Chap. 3], is denoted $S(\mathcal{C})$. The $n$-simplices are sequences $c_{0} \supsetneqq \cdots \supsetneqq c_{n}$ in $\mathcal{C}$ which we denote $\mathbf{c}$. Face maps are inclusion of simplices. We view $S(\mathcal{C})$ as a topological space via the geometric realization. Clearly $G$ acts on $S(\mathcal{C})$ whose orbit space is denoted $[S(\mathcal{C})]$. It is a $\mathrm{CW}$-complex obtained as the geometric realization of the simplicial set $\operatorname{Nr}(\mathcal{C}) / G$ where $\operatorname{Nr}(-)$ is the nerve construction $[\mathbf{3}$, XI.2.1]. By abuse of notation, $[S(\mathcal{C})]$ will also denote the poset of the cells of $[S(\mathcal{C})]$ ordered by inclusion.

As a special case consider the poset $\Delta_{p}(G)$ of the non-trivial $p$-subgroups of a finite group $G$. Note that the isotropy group of an $n$-simplex $\mathbf{P}=\left(P_{0}<\cdots<P_{n}\right)$ in $S\left(\Delta_{p}(G)\right)$ is

$$
N_{G}(\mathbf{P})=\cap_{i=0}^{n} N_{G}\left(P_{i}\right) .
$$

The objects of the poset $\left[S\left(\Delta_{p}(G)\right)\right]$, viewed as a small category, are the $G$-conjugacy classes $[\mathbf{P}]$ of the simplices of $S\left(\Delta_{p}(G)\right)$ and there is a unique morphism $[\mathbf{Q}] \rightarrow[\mathbf{P}]$ if the simplex $\mathbf{Q}$ is conjugate in $G$ to a face of $\mathbf{P}$. There is a functor $\mathcal{N}_{G}:\left[S\left(\Delta_{p}(G)\right)\right] \rightarrow \mathbf{A b}$ defined by Linckelmann in $[\mathbf{9}]$

$$
\mathcal{N}_{G}([\mathbf{P}])=\operatorname{Hom}\left(N_{G}(\mathbf{P}), k^{\times}\right)=\operatorname{Hom}\left(N_{G}(\mathbf{P})_{\mathrm{ab}}, k^{\times}\right) .
$$

Theorem 1.2 of [9] implies that the gluing problem in $\mathcal{F}$ has a solution if we can prove that $H^{1}\left(\left[S\left(\Delta_{p}(K)\right)\right] ; \mathcal{N}_{K}\right)=0$ for all $K=\operatorname{Aut}_{\mathcal{F}}(Q) / \operatorname{Inn}(Q)$ where $Q$ is an $\mathcal{F}$-centric subgroup. Thus, if we can prove that $H^{1}\left(\left[S\left(\Delta_{p}(G)\right)\right] ; \mathcal{N}_{G}\right)=0$ for all finite groups $G$, then the gluing problem has a solution for all fusion systems. The purpose of this note is to show that this programme, suggested by Linckelmann, is not feasible.

Theorem 1.1. Set $G=\Sigma_{p^{2}}$. If $p \geqslant 5$ then $H^{1}\left(\left[S\left(\Delta_{p}(G)\right)\right] ; \mathcal{N}_{G}\right) \neq 0$.

We remark that $\Sigma_{p^{2}}$ appears as an outer $\mathcal{F}$-automorphism group of $Q=\left(C_{p}\right)^{p^{2}}$ in the fusion system of the principal block of $C_{p} \prec \Sigma_{p^{2}}$. We also remark, without proof, that Theorem 1.1 is valid for $p=3$ but it fails if $p=2$. For $p=2$ one observes that $H_{G}^{*}\left(\left|\mathcal{B}_{p}(G)\right| ; \mathcal{H}^{1}\right)=0$, see equation (1), because $\mathcal{H}^{1}$ vanishes on all the orbits of $\left|\mathcal{B}_{p}(G)\right|$. For $p=3$ one has to examine the exact sequence (3) more carefully than we do in Propositions 4.2-4.4.

\section{Subdivision categories and higher limits}

Let $G$ be a finite group. As in the introduction, if $\mathcal{C}$ is a finite $G$-poset, let $S(\mathcal{C})$ denote the associated $G$-simplicial complex and let $[S(\mathcal{C})]$ denote its orbit space. We will denote the set of $n$-simplices of $S(\mathcal{C})$ by $S(\mathcal{C})_{n}$. It is the set of the nondegenerate $n$-simplices of $\operatorname{Nr}(\mathcal{C})$. Thus, the $n$-simplices of $S(\mathcal{C})$ are sequences $\mathbf{c}$ of the form $c_{0} \supsetneqq \cdots \supsetneqq c_{n}$ in $\mathcal{C}$. The faces of $\mathbf{c}$ are its non-empty subsequences.

The space $[S(\mathcal{C})]$ is the geometric realization of the simplicial set $\operatorname{Nr}(\mathcal{C}) / G$ whose set of non-degenerate simplices is $[S(\mathcal{C})]_{n}=S(\mathcal{C})_{n} / G$ which in turn, corresponds to the set of $n$-cells of $[S(\mathcal{C})]$. We obtain a poset, abusively denoted $[S(\mathcal{C})]$, whose objects 
are the $G$-orbits of the simplices of $S(\mathcal{C})$ with an arrow $\left[\mathbf{c}^{\prime}\right] \rightarrow[\mathbf{c}]$ if $\mathbf{c}^{\prime}$ is in the orbit of a face of $\mathbf{c}$. The objects of $[S(\mathcal{C})]$ will be referred to as "simplices".

Given an $n$-simplex $c_{0} \supsetneqq \cdots \supsetneqq c_{n}$ in $S(\mathcal{C})$ where $n \geqslant 1$, we will write $\partial_{i}$ c for the $(n-1)$-simplex obtained by removing $c_{i}$ where $0 \leqslant i \leqslant n$. We obtain face maps

$$
\partial_{i}: S(\mathcal{C})_{n} \rightarrow S(\mathcal{C})_{n-1} \quad \text { and } \quad\left[\partial_{i}\right]:[S(\mathcal{C})]_{n} \rightarrow[S(\mathcal{C})]_{n-1}, \quad(0 \leqslant i \leqslant n)
$$

where $\partial_{i}$ is $G$-equivariant and an $n$-simplex $[\mathbf{c}]$ in $[S(\mathcal{C})]$ gives rise to a map of transitive $G$-sets $[\mathbf{c}] \stackrel{\left[\partial_{i}\right]}{\longrightarrow}\left[\partial_{i} \mathbf{c}\right]$.

Definition 2.1. Fix a commutative $\operatorname{ring} R$. Let $\mathcal{C}$ be a finite $G$-poset and consider a functor $\mathcal{A}:[S(\mathcal{C})] \rightarrow R$-mod. Define a cochain complex $C^{*}(\mathcal{A})$ as follows.

$$
C^{n}(\mathcal{A})=\prod_{[\mathbf{c}] \in[S(\mathcal{C})]_{n}} \mathcal{A}([\mathbf{c}]), \quad \text { and } \quad d: C^{n-1}(\mathcal{A}) \stackrel{\sum_{j=0}^{n}(-1)^{j} d^{j}}{\longrightarrow} C^{n}(\mathcal{A}) .
$$

The homomorphisms $d^{j}: C^{n-1}(\mathcal{A}) \rightarrow C^{n}(\mathcal{A})$ are defined on the $[\mathbf{c}]$-th component of $C^{n}(\mathcal{A})$, where $[\mathbf{c}] \in[S(\mathcal{C})]_{n}$, by the composition

$$
C^{n-1}(\mathcal{A}) \stackrel{\operatorname{proj}}{\longrightarrow} \mathcal{A}\left(\left[\partial_{j} \mathbf{c}\right]\right) \stackrel{\mathcal{A}\left(\left[\partial_{j} \mathbf{c}\right] \preceq[\mathbf{c}]\right)}{\longrightarrow} \mathcal{A}([\mathbf{c}]) .
$$

Lemma 2.2 (cf. [10, Proposition 3.2]). With the notation of Definition 2.1, the cohomology groups of $C^{*}(\mathcal{A})$ are isomorphic to $H^{*}([S(\mathcal{C})] ; \mathcal{A})$.

Proof. For every $n \geqslant 0$ consider the projective functors $P_{n}:[S(\mathcal{C})] \rightarrow \mathbf{A b}$ defined by

$$
P_{n}=\bigoplus_{[\mathbf{c}] \in[S(\mathcal{C})]_{n}} \mathbb{Z} \otimes \operatorname{Mor}_{[S(\mathcal{C})]}([\mathbf{c}],-) .
$$

For every $0 \leqslant j \leqslant n$ there are morphisms $d_{n-1}^{j}: P_{n} \rightarrow P_{n-1}$ which are induced by Yoneda's lemma via the morphisms $\left[\partial_{j} \mathbf{c}\right] \rightarrow[\mathbf{c}]$ for every $[\mathbf{c}] \in[S(\mathcal{C})]_{n}$. Define morphisms $d_{n-1}: P_{n} \rightarrow P_{n-1}$ by $d_{n-1}=\sum_{j=0}^{n}(-1)^{j} d_{n-1}^{j}$. We claim that the resulting

$$
\cdots \rightarrow P_{n} \stackrel{d_{n-1}}{\longrightarrow} P_{n-1} \rightarrow \ldots \stackrel{d_{1}}{\longrightarrow} P_{1} \stackrel{d_{0}}{\longrightarrow} P_{0} \rightarrow \underline{\mathbb{Z}} \quad\left(\text { denoted } P_{\bullet} \rightarrow \underline{\mathbb{Z}}\right)
$$

is a projective resolution of the constant functor $\underline{\mathbb{Z}}$. Indeed, the evaluation of $P_{\bullet}$ at every object $[\mathbf{x}] \in[S(\mathcal{C})]_{n}$ yields the chain complex $C_{*}\left(\Delta^{n} ; \mathbb{Z}\right)$ because the faces of $[\mathbf{x}]$ in $[S(\mathcal{C})]$ generate the standard simplex $\Delta^{n}$. Finally, by Yoneda's Lemma $\operatorname{Hom}\left(P_{\bullet}, \mathcal{A}\right)=C^{*}(\mathcal{A})$ and its homology groups are isomorphic to $\lim ^{*} \mathcal{A}$.

\section{Bredon cohomology}

Throughout this section a space means a simplicial set. Let $G$ be a finite group. A coefficient functor $\mathcal{M}$ for $G$ is a contravariant functor $\{G$-sets $\} \rightarrow \mathbf{A b}$ which turns coproducts of $G$-sets into products of abelian groups. By applying $\mathcal{M}$ to the sets of simplices of a $G$-space $X$, one obtains a cosimplicial abelian group $\mathcal{M}(X)$. The cochain complex associated to $\mathcal{M}(X)$ is denoted $C^{*}(X ; \mathcal{M})$, see $[\mathbf{1 5}, 8.2]$. Its homology groups are called the Bredon cohomology groups $H_{G}^{*}(X ; \mathcal{M})$, see e.g., $[\mathbf{5}, \S 4]$. Note that $C^{n}(X ; \mathcal{M})=\prod_{[\mathbf{x}] \subseteq X} \mathcal{M}([\mathbf{x}])$ where the product runs through the orbits of the $n$ simplices of $X$. 
If $Y$ is a $G$-subspace of $X$ then there is a canonical short exact sequence of cochain complexes

$$
0 \rightarrow C_{G}^{*}(X, Y ; \mathcal{M}) \rightarrow C_{G}^{*}(X ; \mathcal{M}) \rightarrow C_{G}^{*}(Y ; \mathcal{M}) \rightarrow 0
$$

which defines the relative cohomology groups $H_{G}^{*}(X, Y ; \mathcal{M})$ together with the usual long exact sequences. Bredon cohomology is an equivariant cohomology theory, cf. [4]. In particular it turns $G$-homotopy equivalences into isomorphisms and if $X$ is a union of subspaces $Y_{1} \cup Y_{2}$, one has the usual Mayer Vietoris sequence.

The normalized cochain complex $N C^{*}(X ; \mathcal{M})$ is a sub-complex of $C^{*}(X ; \mathcal{M})$ defined by

$$
N C^{n}(X ; M)=\bigcap_{i=0}^{n-1}\left(\operatorname{Ker}\left(C^{n}(X ; \mathcal{M}) \stackrel{s^{i}}{\longrightarrow} C^{n-1}(X ; \mathcal{M})\right)\right),
$$

where $s^{i}$ are the codegeneracy maps of the cosimplicial group $\mathcal{M}(X)$. If $[\mathbf{x}]$ is the orbit of a simplex in $X$ and $s_{i}$ is a degeneracy operator of $X$, it is easy to check that $s_{i}:[\mathbf{x}] \rightarrow\left[s_{i} \mathbf{x}\right]$ is an isomorphism of transitive $G$-sets and in particular $\mathcal{M}([\mathbf{x}])=\mathcal{M}\left(\left[s_{i} \mathbf{x}\right]\right)$. It easily follows that $N C^{n}(X ; \mathcal{M})=\prod_{[\mathbf{x}] \subseteq X} \mathcal{M}([\mathbf{x}])$ where $[\mathbf{x}]$ runs through the orbits of the non-degenerate $n$-simplices of $X$.

It is well known that the inclusion of $N C^{*}(X ; \mathcal{M})$ in $C^{*}(X ; \mathcal{M})$ is a homology equivalence. See $[\mathbf{1 5}, 8.3]$.

Recall that the Borel construction of a $G$-space $U$ is $U \times_{G} E G$ where $E G$ is a contractible space on which $G$ acts freely. If $U=G / K$ then $U \times_{G} E G=B K$ is the classifying space of $K$.

Definition 3.1. Fix a finite group $G$, an abelian group $A$ and an integer $n \geqslant 0$. Define a coefficient functor $\mathcal{H}^{n}$ for $G$ by $\mathcal{H}^{n}(U)=H^{n}\left(U \times_{G} E G ; A\right)$. Observe that $\mathcal{H}^{n}(G / K)=H^{n}(K ; A)$ where $A$ has the trivial action of $K$.

Definition 3.2. Let $\mathcal{C}$ be a finite $G$-poset and let $\mathcal{M}$ be a coefficient system. The underlying set of every object $[\mathbf{c}]$ of $[S(\mathcal{C})]$ is a transitive $G$-set and we define a functor $\mathcal{A}_{\mathcal{M}}:[S(\mathcal{C})] \rightarrow \mathbf{A b}$ by

$$
\mathcal{A}_{\mathcal{M}}([\mathbf{c}])=\mathcal{M}([\mathbf{c}]) .
$$

If $\left[\mathbf{c}^{\prime}\right]$ is a face of $[\mathbf{c}]$, we define $\mathcal{A}_{\mathcal{M}}\left(\left[\mathbf{c}^{\prime}\right] \rightarrow[\mathbf{c}]\right)$ by applying $\mathcal{M}$ to the map $[\mathbf{c}] \rightarrow\left[\mathbf{c}^{\prime}\right]$ of transitive $G$-sets.

By inspection of Definition $2.1, C^{*}\left(\mathcal{A}_{\mathcal{M}}\right) \cong N C_{G}^{*}(|\mathcal{C}| ; \mathcal{M})$ and the next result follows from Lemma 2.2. It has been observed by Słominska [12, p. 116] and by others e.g., Grodal in [6, Theorem 7.3], Linckelmann [10, Proposition 3.5] and Dwyer in [5].

Lemma 3.3. Let $\mathcal{C}$ be a finite $G$-poset and let $\mathcal{M}$ be a coefficient functor for $G$. With the notation of Definition 3.2, $H^{*}\left([S(\mathcal{C})] ; \mathcal{A}_{\mathcal{M}}\right) \cong H_{G}^{*}(|\mathcal{C}| ; \mathcal{M})$.

\section{Proof of Theorem 1.1}

Set $G=\Sigma_{p^{2}}$ and let $\mathcal{C}$ denote the poset $\Delta_{p}(G)$ of the non-trivial $p$-subgroups of $G$. First we observe that $\operatorname{Hom}(K, A)=H^{1}(K ; A)$ for any finite group $K$ and any abelian group $A$. Thus, the functor $\mathcal{N}_{G}:[S(\mathcal{C})] \rightarrow \mathbf{A b}$ defined in the introduction is 
canonically isomorphic to $\mathcal{A}_{\mathcal{H}^{1}}$ as defined in 3.2 and in 3.1 with $A=k^{\times}$where $k$ is an algebraically closed field of characteristic $p$. In light of Lemma 3.3 we need to prove that $H_{G}^{1}\left(\left|\Delta_{p}(G)\right| ; \mathcal{H}^{1}\right) \neq 0$. Consider the $G$-subposet $\mathcal{B}_{p}(G)$ of the nontrivial radical $p$-subgroups of $G$, namely the non-trivial $p$-subgroup $P \leqslant G$ such that $N_{G}(P) / P$ contains no non-trivial normal $p$-subgroup. It is well known that the inclusion $\left|\mathcal{B}_{p}(G)\right| \subseteq\left|\Delta_{p}(G)\right|$ is a $G$-homotopy equivalence, see e.g., [2, Proposition 6.6.5]. Therefore, it remains to prove that

$$
H_{G}^{1}\left(\left|\mathcal{B}_{p}(G)\right| ; \mathcal{H}^{1}\right) \neq 0
$$

The radical $p$-subgroups of the symmetric groups were classified by Alperin and Fong in [1]. In $G=\Sigma_{p^{2}}$ they form the following conjugacy classes:

(R1) The conjugacy class of the Sylow $p$-subgroup $V_{1,1} \stackrel{\text { def }}{=} C_{p} \imath C_{p} \leqslant \Sigma_{p^{2}}$. Its normalizer is $V_{1,1} \rtimes\left(\mathrm{GL}_{1}(p) \times \mathrm{GL}_{1}(p)\right)$ with the diagonal action of $\mathrm{GL}_{1}(p)$ on the base group $\left(C_{p}\right)^{p}$ and the usual action of the second $\mathrm{GL}_{1}(p)$ on $C_{p}$ at the top.

(R2) The conjugacy class of the subgroup $V_{2}=C_{p} \times C_{p}$ embedded in $\Sigma_{p^{2}}$ via its action on itself by translation. Its normalizer is $V_{2} \rtimes \mathrm{GL}_{2}(p)$.

(R3) For every $k=1, \ldots, p$ the conjugacy class of the subgroup $V_{1} \times k$ which is isomorphic to $C_{p}{ }^{\times k}$ as a subgroup of $\Sigma_{p} \times k \leqslant \Sigma_{p^{2}}$. The normalizer of $V_{1}^{\times k}$ is

$$
\left(\left(V_{1} \rtimes \mathrm{GL}_{1}(p)\right)<\Sigma_{k}\right) \times \Sigma_{p(p-k)} .
$$

Definition 4.1. Consider the following subposets of $\mathcal{B}_{p}(G)$.

1. Let $\mathcal{D}_{1}$ be the subposet consisting of the conjugacy class of $V_{1,1}$ and the conjugacy classes of $V_{1}, V_{1}^{\times 2}, \ldots, V_{1}^{\times p}$.

2. Let $\mathcal{V}_{1}$ be the subposet consisting of the conjugacy classes of $V_{1}, V_{1}^{\times 2}, \ldots, V_{1} \times p$.

3. Let $\mathcal{D}_{2}$ be the subposet consisting of the conjugacy classes of $V_{1,1}$ and $V_{2}$.

4. Let $\mathcal{D}_{3}$ be the subposet consisting of the conjugacy class of $V_{1,1}$.

Observe that $V_{2}$ is a transitive subgroup of $\Sigma_{p^{2}}$ so it cannot be conjugate to a subgroup of $V_{1}^{\times k}$ whose orbits have cardinality $p$. Also, $V_{2}$ acts freely so it cannot contain a conjugate of $V_{1}^{\times k}$ since the latter do not act freely on the underlying set of $p^{2}$ elements. We see that up to conjugacy $\mathcal{B}_{p}(G)$ has the form

$$
\left[V_{1}\right]<\left[V_{1}^{\times 2}\right]<\ldots\left[V_{1}^{\times p}\right]<\left[V_{1,1}\right]>\left[V_{2}\right]
$$

and it follows that

$$
\left|\mathcal{B}_{p}(G)\right|=\left|\mathcal{D}_{1}\right| \cup\left|\mathcal{D}_{2}\right|, \quad \text { and } \quad\left|\mathcal{D}_{3}\right|=\left|\mathcal{D}_{1}\right| \cap\left|\mathcal{D}_{2}\right| .
$$

The Mayer Vietoris sequence gives an exact sequence

$$
\cdots \rightarrow H_{G}^{0}\left(\left|\mathcal{D}_{1}\right| ; \mathcal{H}^{1}\right) \oplus H_{G}^{0}\left(\left|\mathcal{D}_{2}\right| ; \mathcal{H}^{1}\right) \rightarrow H_{G}^{0}\left(\left|\mathcal{D}_{3}\right| ; \mathcal{H}^{1}\right) \rightarrow H_{G}^{1}\left(\left|\mathcal{B}_{p}(G)\right| ; \mathcal{H}^{1}\right) \rightarrow \ldots
$$

For what follows, it will be convenient to denote

$$
L=\operatorname{Hom}\left(\mathrm{GL}_{1}(p), k^{\times}\right) \cong \mathbb{F}_{p}^{\times} .
$$

Proposition 4.2. $H_{G}^{0}\left(\left|\mathcal{D}_{3}\right| ; \mathcal{H}^{1}\right) \cong L \times L$ and $H_{G}^{* \geqslant 1}\left(\left|\mathcal{D}_{3}\right| ; \mathcal{H}^{1}\right)=0$. 
Proposition 4.3. $H_{G}^{0}\left(\left|\mathcal{D}_{2}\right| ; \mathcal{H}^{1}\right) \cong L$ and $H_{G}^{* \geqslant 1}\left(\left|\mathcal{D}_{2}\right| ; \mathcal{H}^{1}\right)=0$.

Proposition 4.4. $H_{G}^{0}\left(\left|\mathcal{D}_{1}\right| ; \mathcal{H}^{1}\right) \cong C_{2}$ and $H_{G}^{* \geqslant 1}\left(\left|\mathcal{D}_{1}\right| ; \mathcal{H}^{1}\right)=0$.

Propositions 4.2-4.4 together with the exact sequence (3) immediately imply (1) because by hypothesis $p \geqslant 5$, whence $|L| \geqslant 4$.

Recall that $k$ has characteristic $p$. Therefore the kernel of any group homomorphism $H \rightarrow k^{\times}$contains the commutator subgroup of $H$ and any $p$-subgroup of $H$. We will use this fact repeatedly.

Proof of Proposition 4.2. Since $\mathcal{D}_{3}$ is a single orbit of $G$ with isotropy group $N_{G}\left(V_{1,1}\right)$ it follows from $(\mathbf{R} 1)$ that $H_{G}^{*}\left(\left|\mathcal{D}_{3}\right| ; \mathcal{H}^{1}\right)=\operatorname{Hom}\left(N_{G}\left(V_{1,1}\right), k^{\times}\right)=L \times L$.

Proof of Proposition 4.3. Since $\left|\mathcal{B}_{p}(G)\right|$ is $G$-equivalent to $\left|\Delta_{p}(G)\right|$, Symond's resolution of Webb's conjecture in $[\mathbf{1 4}]$ shows that the orbit space $\left|\mathcal{B}_{p}(G)\right| / G$ is contractible. But (2) shows that $\left|\mathcal{B}_{p}(G)\right| / G=\left(\left|\mathcal{D}_{1}\right| / G\right) \vee\left(\left|\mathcal{D}_{2}\right| / G\right)$. It follows that the CW-complex $\left|D_{2}\right| / G$, namely $\left[S\left(\mathcal{D}_{2}\right)\right]$, is contractible and since it is 1-dimensional with two 0 -simplices $\left[V_{2}\right]$ and $\left[V_{1,1}\right]$, the poset $\left[S\left(\mathcal{D}_{2}\right)\right]$ must have the form

$$
\left[V_{2}\right] \rightarrow\left[V_{2}<V_{1,1}\right] \leftarrow\left[V_{1,1}\right] .
$$

Now, $V_{2} \leqslant V_{1,1}=C_{p} 2 C_{p}$ is generated by the copy of $C_{p}$ at the top and the diagonal copy of $C_{p}$ in the base group $C_{p} \times \cdots \times C_{p}$ which is the centre of $V_{1,1}$. One easily deduces from $(\mathbf{R 1})$ and $(\mathbf{R 2})$ that $N_{G}\left(V_{2}<V_{1,1}\right) / N_{V_{1,1}}\left(V_{2}\right) \cong \mathrm{GL}_{1}(p)^{2}$ as a diagonal subgroup of $\mathrm{GL}_{2}(p)$. With the notation of Definition 3.2 we have

$$
\mathcal{A}_{\mathcal{H}^{1}}\left(\left[V_{2}<V_{1,1}\right]\right) \cong \operatorname{Hom}\left(\mathrm{GL}_{1}(p)^{2}, k^{\times}\right) \cong \mathcal{A}_{\mathcal{H}^{1}}\left(\left[V_{1,1}\right]\right),
$$

and $\mathcal{A}_{\mathcal{H}^{1}}\left(\left[V_{2}\right]\right)=\operatorname{Hom}\left(\mathrm{GL}_{2}(p), k^{\times}\right) \cong L$ because $\mathrm{GL}_{2}(p)_{\mathrm{ab}}=\mathbb{F}_{p}^{\times}$. By Lemma 3.3 , the groups $H_{G}^{*}\left(\left|\mathcal{D}_{2}\right| ; \mathcal{H}^{1}\right)$ are isomorphic to $H^{*}\left(\left[S\left(\mathcal{D}_{2}\right)\right] ; \mathcal{A}_{\mathcal{H}^{1}}\right)$, namely to the derived functors of the diagram $L \stackrel{\Delta}{\longrightarrow} L \times L \stackrel{\text { id }}{\longleftarrow} L \times L$. This completes the proof.

Lemma 4.5. The inclusion $\mathcal{V}_{1} \subseteq \mathcal{D}_{1}$, see Definition 4.1, induces a G-equivariant homotopy equivalence $\left|\mathcal{V}_{1}\right| \rightarrow\left|\mathcal{D}_{1}\right|$.

Proof. Given a subgroup $P$ of $G$ let $\delta_{1}(P)$ denote the subgroup of $P$ generated by all the permutations $g \in P$ whose support contains at most $p$ elements. Observe that $\delta_{1}$ is invariant under conjugation, namely $\delta_{1}\left(g P g^{-1}\right)=g \delta_{1}(P) g^{-1}$. By inspection $\delta_{1}\left(V_{1}^{\times k}\right)=V_{1}^{\times k}$ and $\delta_{1}\left(V_{1,1}\right)=V_{1}^{\times p}$. We obtain a $G$-equivariant morphism of posets $\delta_{1}: \mathcal{D}_{1} \rightarrow \mathcal{V}_{1}$. Clearly, $\left|\delta_{1}\right| \circ i_{\left|\mathcal{V}_{1}\right|}^{\left|\mathcal{D}_{1}\right|}=\operatorname{Id}_{\left|\mathcal{V}_{1}\right|}$. The inclusions $\delta_{1}(P) \leqslant P$ give a $G$ equivariant homotopy $i_{\left|\mathcal{V}_{1}\right|}^{\left|\mathcal{D}_{1}\right|} \circ\left|\delta_{1}\right| \simeq \operatorname{Id}_{\left|\mathcal{D}_{1}\right|}$, cf. $[\mathbf{1 1}, 1.3]$. The result follows.

We leave the following result as an easy exercise for the reader.

Lemma 4.6. Let $K$ be a finite group, fix an integer $n \geqslant 1$ and set $G_{n}=K \backslash \Sigma_{n}$. Then $\left(G_{n}\right)_{\mathrm{ab}} \cong K_{\mathrm{ab}} \times\left(\Sigma_{n}\right)_{\mathrm{ab}}$. The restriction of $G_{n} \rightarrow\left(G_{n}\right)_{\mathrm{ab}}$ to any one of the factors $K$ of $K^{n} \leqslant G_{n}$ is the canonical projection $K \rightarrow K_{\mathrm{ab}}$ and the restriction of $G_{n} \rightarrow\left(G_{n}\right)_{\mathrm{ab}}$ to $\Sigma_{n}$ is the projection onto $\left(\Sigma_{n}\right)_{\mathrm{ab}}$.

If $n, m \geqslant 1$ then $G_{n} \times G_{m} \leqslant G_{n+m}$. The resulting $\left(G_{n}\right)_{\mathrm{ab}} \times\left(G_{m}\right)_{\mathrm{ab}} \rightarrow\left(G_{n+m}\right)_{\mathrm{ab}}$ is induced by the fold map $K_{\mathrm{ab}} \times K_{\mathrm{ab}} \rightarrow K_{\mathrm{ab}}$ and by $\left(\Sigma_{n}\right)_{\mathrm{ab}} \times\left(\Sigma_{m}\right)_{\mathrm{ab}} \rightarrow\left(\Sigma_{n+m}\right)_{\mathrm{ab}}$. 
Notation 4.7. The following non-standard description of the $(n-1)$-simplex $\Delta^{n-1}$ will be used throughout. The $r$-simplices of $\Delta^{n-1}$ are sequences $i_{0}<\cdots<i_{r}$ where $1 \leqslant i_{0}, \ldots, i_{r} \leqslant n$. Face maps are obtained by inclusion of sequences. (The usual convention is $0 \leqslant i_{0}, \ldots, i_{r} \leqslant n-1$.)

Proof of Proposition 4.4. In light of Lemma 4.5 and Lemma 3.3, we must prove that $H_{G}^{*}\left(\left[S\left(\mathcal{V}_{1}\right)\right] ; \mathcal{A}_{\mathcal{H}^{1}}\right) \cong C_{2}$.

The high transitivity of the symmetric groups and the description of $N_{G}\left(V_{1}^{\times k}\right)$ in (R3) imply that every $r$-simplex of $S\left(\mathcal{V}_{1}\right)$ is conjugate in $G$ to a simplex of the form $V_{1}^{\times i_{0}}<\cdots<V_{1}^{\times i_{r}}$ where $1 \leqslant i_{0}<\cdots<i_{r} \leqslant p$. With the notation of 4.7 we see that $\left[S\left(\mathcal{V}_{1}\right)\right]=\Delta^{p-1}$.

For any group $K$ let $\widehat{K}$ denote the abelian group $\operatorname{Hom}\left(K, k^{\times}\right)$. Let $N$ denote the normalizer of $C_{p}$ in $\Sigma_{p}$. Thus, $N=C_{p} \rtimes \mathrm{GL}_{1}(p)$ and observe that $\mathrm{GL}_{1}(p) \leqslant \Sigma_{p}$ is generated by an odd permutation, in fact a cycle of even length ( $p$ is odd). Set

$$
L=\widehat{N}=\operatorname{Hom}\left(N, k^{\times}\right)=\operatorname{Hom}\left(\mathrm{GL}_{1}(p), k^{\times}\right) \cong C_{p-1} .
$$

Consider the following functor $\Phi:\left(\Delta^{p-1}\right)^{\text {op }} \rightarrow\{$ Groups $\}$. On objects

$$
\left.\Phi\left(i_{0}<\cdots<i_{r}\right)=\left(\prod_{t=0}^{r} N<\Sigma_{i_{t}-i_{t-1}}\right) \times \Sigma_{p^{2}-i_{r} p}, \quad \text { (by convention } i_{-1}=0\right) .
$$

For an $r$-simplex $\mathbf{i}$ and for $0 \leqslant j \leqslant r$, the effect of $\Phi(\mathbf{i}) \rightarrow \Psi\left(\partial_{j} \mathbf{i}\right)$ is induced by the inclusions

$$
\begin{array}{ll}
\left(N<\Sigma_{i_{j}-i_{j-1}}\right) \times\left(N<\Sigma_{i_{j+1}-i_{j}}\right) \leqslant\left(N<\Sigma_{i_{j+1}-i_{j-1}}\right) & \text { if } 0 \leqslant j<r \\
\left(N<\Sigma_{i_{r}-i_{r-1}}\right) \times \Sigma_{p\left(p-i_{r}\right)} \leqslant \Sigma_{p\left(p-i_{r-1}\right)} & \text { if } j=r .
\end{array}
$$

Inspection of $(\mathbf{R 3})$ shows that $\mathcal{A}_{\mathcal{H}^{1}}=\widehat{\Phi}$, namely $\mathcal{A}_{\mathcal{H}^{1}}=\operatorname{Hom}\left(\Phi, k^{\times}\right)$. Having identified $\left[S\left(\mathcal{V}_{1}\right)\right]$ with $\Delta^{p-1}$, it remains to prove that

$$
H^{*}\left(\Delta^{p-1} ; \widehat{\Phi}\right) \cong C_{2} \text {. }
$$

Consider the following functor $\Psi: \Delta^{p-1} \rightarrow \mathbf{A b}$ defined by

$$
\left.\Psi\left(i_{0}<\cdots<i_{r}\right)=\left(\prod_{t=0}^{r} N<\Sigma_{i_{t}-i_{t-1}}\right) \times\left(N<\Sigma_{p-i_{r}}\right), \quad \text { (by convention } i_{-1}=0\right) .
$$

It is a subfunctor of $\Phi$ via the inclusions $N<\Sigma_{p-i_{r}} \leqslant \Sigma_{p\left(p-i_{r}\right)}$. We obtain a morphism of functors $\widehat{\Phi} \rightarrow \widehat{\Psi}$ of abelian groups. Our goal now is to prove that it is a monomorphism and to calculate its cokernel. Fix an $r$-simplex $\mathbf{i}=\left(i_{0}<\cdots<i_{r}\right)$ in $\Delta^{p-1}$ and consider $\widehat{\Phi}(\mathbf{i}) \rightarrow \widehat{\Psi}(\mathbf{i})$. Note that $\left(\Sigma_{n}\right)_{\mathrm{ab}}=C_{2}$ if $n \geqslant 2$ and that if $H \leqslant \Sigma_{n}$ contains an odd permutation then $H_{\mathrm{ab}} \rightarrow\left(\Sigma_{n}\right)_{\mathrm{ab}}$ is surjective.

Case (a). If $i_{r}=p$ then $\Sigma_{p^{2}-i_{r} p}$ and $N \backslash \Sigma_{p-i_{r}}$ are the trivial group and therefore $\widehat{\Phi}(\mathbf{i}) \rightarrow \widehat{\Psi}(\mathbf{i})$ is an isomorphism.

Case (b). If $i_{r}=p-1$ then $N<\Sigma_{p-i_{r}}=N$ and $\Sigma_{p\left(p-i_{r}\right)}=\Sigma_{p}$. Since $N=C_{p} \rtimes$ $C_{p-1}$ contains an odd permutation, by applying $\operatorname{Hom}\left(-, k^{\times}\right)$to the inclusion $N \leqslant \Sigma_{p}$ we obtain the monomorphism $C_{2} \rightarrow L$ and therefore $\widehat{\Phi}(\mathbf{i}) \rightarrow \widehat{\Psi}(\mathbf{i})$ is injective with cokernel $L / C_{2}$.

Case (c). Assume that $i_{r} \leqslant p-2$. The inclusion of $N^{p-i_{r}} \leqslant \Sigma_{p\left(p-i_{r}\right)}$ contains odd permutations. Since $p$ is odd, also the diagonal inclusion $\Sigma_{p-i_{r}} \leqslant \Sigma_{p\left(p-i_{r}\right)}$ contains 
odd permutations. By Lemma 4.6 the induced map $\widehat{\Sigma_{p\left(p-i_{r}\right)}} \rightarrow N \widehat{\Sigma_{p-i_{r}}}$ is the diagonal inclusion $C_{2} \rightarrow L \oplus C_{2}$ into $C_{2} \oplus C_{2}$. It follows that $\widehat{\Phi}(\mathbf{i}) \rightarrow \widehat{\Psi}(\mathbf{i})$ is injective with cokernel $L$.

We obtain a short exact sequence of functors $\Delta^{p-1} \rightarrow \mathbf{A b}$

$$
0 \rightarrow \widehat{\Phi} \rightarrow \widehat{\Psi} \rightarrow \Gamma \rightarrow 0,
$$

where the functor $\Gamma$ has the form

$$
\Gamma(\mathbf{i})= \begin{cases}0 & \text { if } i_{r}=p \\ L / C_{2} & \text { if } i_{r}=p-1 \\ L & \text { if } i_{r} \leqslant p-2 .\end{cases}
$$

By Lemma 4.6, $\Gamma(\mathbf{j}) \rightarrow \Gamma(\mathbf{i})$ are induced by the quotient maps $L \rightarrow L / C_{2} \rightarrow 0$.

Let $\Gamma^{\prime}, \Gamma^{\prime \prime}: \Delta^{p-1} \rightarrow \mathbf{A b}$ be the functors defined for objects $\mathbf{i}=\left(i_{0}<\cdots<i_{r}\right)$ by

$$
\Gamma^{\prime}(\mathbf{i})=\left\{\begin{array}{ll}
L & \text { if } 1 \leqslant i_{r} \leqslant p-1 \\
0 & \text { if } i_{r}=p
\end{array} \quad \Gamma^{\prime \prime}(\mathbf{i})= \begin{cases}C_{2} & \text { if } i_{r}=p-1 \\
0 & \text { if } i_{r} \neq p-1 .\end{cases}\right.
$$

Face maps $\mathbf{i} \subseteq \mathbf{j}$ induce either the identity or the trivial homomorphisms $\Gamma^{\prime}(\mathbf{i}) \rightarrow \Gamma^{\prime}(\mathbf{j})$ and $\Gamma^{\prime \prime}(\mathbf{i}) \rightarrow \Gamma^{\prime \prime}(\mathbf{j})$. We get a short exact sequence of functors

$$
0 \rightarrow \Gamma^{\prime \prime} \rightarrow \Gamma^{\prime} \rightarrow \Gamma \rightarrow 0 .
$$

We view $\Delta^{p-2}$ as the $(p-1)$ st face of $\Delta^{p-1}$, that is, $\Delta^{p-2}$ consist of the simplices $\mathbf{i}=\left(i_{0}<\cdots<i_{r}\right)$ of $\Delta^{p-1}$ with $i_{r} \leqslant p-1$. Similarly $\Delta^{p-3}$ is the $(p-2)$ nd face of $\Delta^{p-2}$. Thus, $\Delta^{p-3}$ is the subcomplex of $\Delta^{p-1}$ of the simplices $\mathbf{i}$ with $i_{r} \leqslant p-2$. At this point we should recall that $p \geqslant 5$.

By inspection of Definition 2.1 we see that $C^{*}\left(\Gamma^{\prime \prime}\right)$ is isomorphic to the cochain complex $C^{*}\left(\Delta^{p-2}, \Delta^{p-3} ; C_{2}\right)$ of the relative simplicial complex $\left(\Delta^{p-2}, \Delta^{p-3}\right)$. Since $p \geqslant 5$, the contractibility of the standard simplices and Lemma 2.2 imply that

$$
H^{*}\left(\Delta^{p-1} ; \Gamma^{\prime \prime}\right) \cong H^{*}\left(\Delta^{p-2}, \Delta^{p-3} ; C_{2}\right)=0 .
$$

The acyclicity of $\Gamma^{\prime \prime}$ now shows that $\Gamma^{\prime} \rightarrow \Gamma$ induces an isomorphism

$$
H^{*}\left(\Delta^{p-1} ; \Gamma^{\prime}\right) \stackrel{\cong}{\longrightarrow} H^{*}\left(\Delta^{p-1} ; \Gamma\right) .
$$

By Lemma 4.6 we see that $\widehat{\Psi}: \Delta^{p-1} \rightarrow \mathbf{A b}$ has the following form

$$
\widehat{\Psi}\left(i_{0}<\cdots<i_{r}\right)=\left(\prod_{t=0}^{r} L \times \widehat{\Sigma_{i_{t}-i_{t-1}}}\right) \times \begin{cases}0 & \text { if } i_{r}=p \\ L \times \widehat{\Sigma_{p-i_{r}}} & \text { if } i_{r}<p .\end{cases}
$$

We obtain a constant subfunctor $\Psi^{\prime}(\mathbf{i})=L$ of $\widehat{\Psi}$ via the diagonal inclusion and it is easy to check that the following square commutes

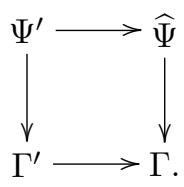

By inspection of Definition 2.1, there are isomorphisms $C^{*}\left(\Psi^{\prime}\right) \cong C^{*}\left(\Delta^{p-1} ; L\right)$ and $C^{*}\left(\Gamma^{\prime}\right) \cong C^{*}\left(\Delta^{p-2} ; L\right)$. The map $\Psi^{\prime} \rightarrow \Gamma^{\prime}$ gives rise to the map of cochain complexes 
induced by $\Delta^{p-2} \subseteq \Delta^{p-1}$. We deduce from Lemma 2.2 and the contractibility of the standard simplices that $\Psi^{\prime} \rightarrow \Gamma^{\prime}$ induces an isomorphism

$$
H^{*}\left(\Delta^{p-1} ; \Psi^{\prime}\right) \cong H^{*}\left(\Delta^{p-1} ; \Gamma^{\prime}\right) \cong \begin{cases}L & \text { if } *=0 \\ 0 & \text { if } *=0\end{cases}
$$

The commutative square above, together with (6) and (7) imply that $\widehat{\Psi} \rightarrow \Gamma$ induces an epimorphism $H^{*}\left(\Delta^{p-1} ; \widehat{\Psi}\right) \rightarrow H^{*}\left(\Delta^{p-1} ; \Gamma\right)$. By $(6)$ and $(7)$ and the long exact sequence associated to (5), the proof of (4), whence the proof of this proposition, will be complete if we prove that $H^{*}\left(\Delta^{p-1} ; \widehat{\Psi}\right) \cong L \oplus C_{2}$ (cohomology concentrated in degree 0$)$.

Set $K=N \backslash \Sigma_{p}$ and let it act on the poset $\Omega$ of the non-empty subsets of $\{1, \ldots, p\}$ via the projection onto $\Sigma_{p}$. One easily checks that $[S(\Omega)]=\Delta^{p-1}$ and that, by choosing appropriate representatives, the isotropy groups of the $r$-simplices of $S(\Omega)$ are

$$
\operatorname{Iso}_{K}\left(i_{0}<\cdots<i_{r}\right)=\Psi\left(i_{0}<\cdots<i_{r}\right) .
$$

Thus, if $\mathcal{H}_{K}^{1}$ is the coefficient functor for $K$ defined in 3.1 with $A=k^{\times}$, we see that $C^{*}(\widehat{\Psi}) \cong C^{*}\left(\mathcal{A}_{\mathcal{H}_{K}^{1}}\right)$, whence by Lemma 3.3 ,

$$
H^{*}\left(\Delta^{p-1} ; \widehat{\Psi}\right) \cong H^{*}\left([S(\Omega)] ; \mathcal{A}_{\mathcal{H}_{K}^{1}}\right) \cong H_{K}^{*}\left(|\Omega| ; \mathcal{H}_{K}^{1}\right) .
$$

Now, $|\Omega|$ is $K$-equivalent to a point because $\{1, \ldots, p\}$ is a maximal element of $\Omega$ fixed by $K$. Therefore $H_{K}^{*}\left(|\Omega| ; \mathcal{H}_{K}^{1}\right) \cong \mathcal{H}_{K}^{1}(\mathrm{pt})=\widehat{N \imath \Sigma_{p}}=L \oplus C_{2}$ by Lemma 4.6. This completes the proof.

\section{References}

[1] J. L. Alperin, P. Fong, Weights for symmetric and general linear groups. $J$. Algebra 131 (1990), no. 1, 2-22.

[2] D. J. Benson, Representations and cohomology. II. Cohomology of groups and modules. Cambridge Studies in Advanced Mathematics, 31. Cambridge University Press, Cambridge, 1991.

[3] A. K. Bousfield, D. M. Kan, Homotopy limits, completions and localizations. Lecture Notes in Mathematics, Vol. 304. Springer-Verlag, Berlin-New York, 1972.

[4] Glen E. Bredon, Equivariant cohomology theories. Lecture Notes in Mathematics, No. 34. Springer-Verlag, Berlin-New York 1967

[5] W. G. Dwyer, Sharp homology decompositions for classifying spaces of finite groups. Group representations: cohomology, group actions and topology (Seattle, WA, 1996), 197-220, Proc. Sympos. Pure Math., 63, Amer. Math. Soc., Providence, RI, 1998.

[6] Jesper Grodal, Higher limits via subgroup complexes. Ann. of Math. (2) 155 (2002), no. 2, 405-457.

[7] Reinhard Knörr, Geoffrey R. Robinson, Some remarks on a conjecture of Alperin. J. London Math. Soc. (2) 39 (1989), no. 1, 48-60. 
[8] Burkhard Külshammer, Lluís Puig, Extensions of nilpotent blocks. Invent. Math. 102 (1990), no. 1, 17-71.

[9] Markus Linckelmann, On $H^{*}\left(\mathcal{C} ; k^{\times}\right)$for fusion systems. Homology, Homotopy and Applications 11(1), 203-218.

[10] Markus Linckelmann, Alperin's weight conjecture in terms of equivariant Bredon cohomology. Math. Z. 250 (2005), no. 3, 495-513.

[11] Daniel Quillen, Homotopy properties of the poset of nontrivial $p$-subgroups of a group. Adv. in Math. 28 (1978), no. 2, 101-128.

[12] Jolanta Słomińska, Some spectral sequences in Bredon cohomology. Cahiers Topologie Géom. Différentielle Catég. 33 (1992), no. 2, 99-133.

[13] Edwin H. Spanier, Algebraic topology. McGraw-Hill Book Co., New YorkToronto, Ont.-London 1966

[14] Peter Symonds, The orbit space of the $p$-subgroup complex is contractible. Comment. Math. Helv. 73 (1998), no. 3, 400-405.

[15] Weibel, Charles A. An introduction to homological algebra. Cambridge Studies in Advanced Mathematics, 38. Cambridge University Press, Cambridge, 1994.

Assaf Libman a.libman@abdn.ac.uk

Mathematics Department, University of Aberdeen, Fraser Noble building, King's College, Aberdeen, AB24 3UE, U.K. 